\title{
O PERFIL DE UM GRUPO DE PESSOAS HIPERTENSAS DE ACORDO COM CONHECIMENTO E GRAVIDADE DA DOENÇA
}

\section{THE PROFILE OF HYPERTENSIVE PATIENTS RELATED TO AWARENESS AND LEVEL OF HYPERTENSION}

\author{
Angela M.G. Pierin* \\ Décio Mion $J r^{* *}$ \\ Júlia T Fukushima*** \\ Alessandro R. Pinto**** \\ Marcia M. Kaminaga****
}

PIERIN, AMG et al. O perfil de um grupo de pessoas hipertensas de acordo com conhecimento e gravidade da doença. Rev Esc Enf USP, v.35, n. 1, p. 11-8, mar. 2001.

\section{RESUMO}

Foram entrevistados 205 hipertensos em tratamento ambulatorial para avaliar o papel do perfil bio-social no conhecimento e grau de gravidade da doença. As características da população foram: $72 \%$ mulheres, $63 \%$ brancos, $78 \%$ com mais de 40 anos, 60\% casados, 68\% com baixa escolaridade, 41\% com renda de 1 a 3 salários, 75\% com peso elevado, 76\% não fumantes, $89 \%$ sem atividade física regular, e das mulheres 48\% já tinham usado hormônios anticoncepcionais. A análise evidenciou que a ausência de conhecimento se associou com sexo masculino, idade entre 20 e 40 anos, viúvo, não branco e peso normal. Pressão arterial mais elevada (diastólica> $110 \mathrm{~mm} \mathrm{Hg)} \mathrm{se} \mathrm{associou} \mathrm{com} \mathrm{mais} \mathrm{de} 60$ anos, não casado, acima do peso, baixa escolaridade, baixa renda, com mais de 5 anos de hipertensão e já ter feito tratamento anterior.

PALAVRAS-CHAVE: Hipertensão pressão arterial. Perfil dos hipertensos.

\section{ABSTRACT}

We interviewed 205 hypertensives to characterize social demographic data, life style, history and awareness of hypertension. Most patients were female (72\%), white (63\%), over 40 years old (78\%), married (60\%), and had low level of education (68\%). Their family income was lower than US\$ 3000 a year (41\%). In terms of weight, 75\% were classified as overweight, $76 \%$ were non smoker, and $89 \%$ did not do any regular physical activity. The profile of the hypertensives who were unaware of the disease was: male, 20-40 years old, widow, not white, and non-obese. The profile of the hypertensives who had high level of hypertension (diastólic $110 \mathrm{~mm} \mathrm{Hg}$ ) was older than 60 years old, not married, overweight, low level of education, low income, with more than 5 years of hypertension, and previously treated.

KEYWORDS: Hypertension blood pressure. Profile of hypertensive.

\section{INTRODUÇÃO}

Um dos maiores desafios no controle da hipertensão arterial é conseguir a adesão dos hipertensos para garantir o sucesso do tratamento a longo prazo. Estudos têm evidenciado que vários fatores podem influenciar a adesão ao tratamento $(1,2)$ destacando-se dentre eles as características bio-sociais e conhecimento sobre a doença e tratamento.

A hipertensão arterial é uma doença altamente prevalente, acometendo cerca de 15 a $20 \%$ a população adulta(3,4) podendo atingir índices mais elevados, de até $50 \%$, nas pessoas idosas $(5,6)$. O tratamento

adequado com o controle dos níveis tensionais, reduz tanto a mortalidade, quanto a morbidade associadas à doença. Portanto, considera-se de suma importância que os hipertensos sigam o tratamento proposto, o que não é tarefa fácil, apesar da efetividade das medidas farmacológicas e não farmacológicas.

A descontinuidade do tratamento é um problema observado na maioria das doenças crônicas que requerem tratamento a longo prazo ou por toda vida. Vários determinantes, isoladamente ou associados, atuam na problemática da adesão.

\footnotetext{
* Professora Livre Docente da Escola de Enfermagem da USP.

** Professor Livre Docente, chefe da Unidade de Hipertensão do Hospital das Clínicas da FM USP.

*** Mestre em estatística

**** Alunos de,graduação da Escola de Enfermagem da USP, bolsistas de iniciação Científica CNPq
} 
Vários estudos têm examinado os elementos intervenientes na adesão ao tratamento. Muitas destas pesquisas têm enfocado as características pessoais e demográficas dos hipertensos; seus conhecimentos; valores, crenças; experiências vividas e expectativas; influências sociais, como suporte familiar; barreiras encontradas, como a falta de recursos; e dificuldad $\backslash$ es para seguir o tratamento $(7,8,9)$. A complexidade do regime terapêutico e efeitos indesejáveis das drogas tendem a dificultar, enquanto que o bom relacionamento do hipertenso com o médico e demais membros da equipe de saúde, pode beneficiar o processo de adesão ao tratamento(1)

Portanto, considera-se de suma importância que para a implementação da assistência aos hipertensos deva-se conhecer as características inerentes ao paciente que possam influenciar na vivência com a doença e tratamento. Face a estas colocações, o presente estudo tem como objetivos:

1) delinear o perfil de um grupo de pessoas hipertensas em relação a variáveis bio-sociais, hábitos de vida, antecedentes de hipertensão arterial e conhecimentos sobre a doença; e

2) avaliar interrelações entre as variáveis identificadas no perfil da população com o conhecimento e nivel de gravidade da doença.

\section{MATERIAL E MÉTODO}

Trata-se de um estudo descritivo realizado em um serviço ambulatorial de um hospital de ensino da cidade de São Paulo que atende pessoas hipertensas.

Foram entrevistados todos os pacientes em um periodo de quatro meses, perfazendo um total de 205 pessoas, com idade acima de 18 anos; de ambos os sexos; atendidos pela primeira vez no serviço e que concordaram em participar do estudo. Inicialmente, os pacientes foram esclarecidos sobre os objetivos da pesquisa e só após a sua anuência iniciou-se a entrevista para coleta dos dados. Os dados apresentados no presente estudo fizeram parte de um projeto maior, devidamente aprovado pelo Comitê de ética da instituição.

Para análise dos dados, considerou-se hipertensão valores acima ou igual a $140 \mathrm{~mm} \mathrm{Hg}$ para a pressão sistólica e, ou $90 \mathrm{~mm} \mathrm{Hg}$ para a diastólica. Quando os valores das pressões sistólica e diastólica se situaram em diferentes faixas de hipertensão considerou-se aquela de maior valor. Na análise das diferentes categorias de hipertensão, considerou-se a pressão diastólica, uma vez que esta representou a real prevalência de hipertensão nas pessoas em estudo. Os niveis de hipertensão considerados para a pressão diastólica foram: $<90 \mathrm{~mm}$ Hg - normal; leve - 90-99 mm Hg; moderada -100-109 $\mathrm{mm} \mathrm{Hg}$; grave - 110-120 mm Hg; e muito grave - >120 mm Hg. Também utilizou-se os niveis de hipertensão considerando a pressão diastólica para análise do perfil dos pacientes em estudo relacionados ao conhecimento sobre a doença e gravidade da hipertensão arterial.

As categorias de índice de massa corpórea foram divididas da seguinte maneira: menor que 25 - normal; 25,0-29,9 - sobrepeso; maior ou igual a 30,- obesidade.

Pára a coleta de dados utilizou-se formulário específico (Anexo 1) incluindo variáveis bio sociais, hábitos de vida, história e conhecimento sobre a doença.

Para a caracterização do perfil dos hipertensos estudados 'em relação ao conhecimento sobre a doença e nível da pressão arterial, utilizou-se análise estatística através do método de análise de correspondência.

\section{RESULTADOS}

\subsection{Características sócio-demográficas dos hipertensos estudados}

Tabela 1 - Distribuição do $n^{\circ}$ e \% dos características sócio-demográficas dos hipertensos em estudo. São Paulo, 1999.

\begin{tabular}{|c|c|c|}
\hline Características & $\mathrm{N}$ & $\%$ \\
\hline \multicolumn{3}{|l|}{ Sexo } \\
\hline Masculino & 57 & 28 \\
\hline Feminino & 148 & 72 \\
\hline \multicolumn{3}{|l|}{ Idade (anos) } \\
\hline$<20$ & 3 & 1 \\
\hline $21-40$ & 42 & 21 \\
\hline $41-60$ & 116 & 57 \\
\hline$>60$ & 44 & 22 \\
\hline \multicolumn{3}{|l|}{ Cor } \\
\hline Branco & 130 & 63 \\
\hline Não Branco & 75 & 37 \\
\hline \multicolumn{3}{|l|}{ Estado Civil } \\
\hline Casado & 126 & 61 \\
\hline Solteiro & 29 & 14 \\
\hline Viúvo & 38 & 19 \\
\hline Separado & 12 & 06 \\
\hline \multicolumn{3}{|l|}{ Escolaridade } \\
\hline Lê/Escreve & 14 & 07 \\
\hline Analfabeto & 25 & 12 \\
\hline $1^{\circ}$ grau completo & 14 & 07 \\
\hline $1^{\circ}$ grau incompleto & 114 & 55 \\
\hline $2^{\circ}$ grau completo & 21 & 10 \\
\hline $2^{\circ}$ grau incompleto & 9 & 05 \\
\hline Superior & 08 & 04 \\
\hline \multicolumn{3}{|l|}{ Renda (sal. minimo) } \\
\hline 1 a 3 & 84 & 41 \\
\hline $4 a 5$ & 71 & 35 \\
\hline$>5$ & 50 & 24 \\
\hline \multirow{2}{*}{\multicolumn{3}{|c|}{$\begin{array}{l}\text { Índice de Massa } \\
\text { Corporal } \\
\text { (peso/altura2) }\end{array}$}} \\
\hline & & \\
\hline Normal & 52 & 25 \\
\hline Sobrepeso & 81 & 40 \\
\hline Obeso & 72 & 35 \\
\hline \multicolumn{3}{|l|}{ Fatores de risco } \\
\hline Sedentarismo & 182 & 89 \\
\hline Uso anticoncepcional & 71 & 48 \\
\hline Hábito de fumar & 51 & 25 \\
\hline Uso bebida alcoólica & 06 & 03 \\
\hline
\end{tabular}


Os dados da Tabela 1 mostram que a maioria da população estudada pertencia ao sexo feminino $(72 \%)$, apresentava idade na faixa de 41 a 60 anos $(57 \%)$ com média \pm desvio padrão de $53 \pm 13$ anos, cor branca (63\%), estado civil casado (60\%), e com 10 grau de escolaridade (62\%), na maioria incompleto $(89 \%)$. A renda salarial se concentrou nas faixas de la $3(41 \%)$ e 4 a $5(35 \%)$ salários mínimos. Quanto ao indice de massa corporal $25 \%$ estavam na faixa de normalidade, $40 \%$ na faixa de sobrepeso e $35 \%$ dos pacientes eram obesos, portanto, $75 \%$ das pessoas estudadas apresentaram índice de massa corporal acima da normalidade.O

hábito de fumar foi referido por apenas 25\% dos hipertensos e quase a totalidade referiu não fazer uso de bebida alcoólica. (97\%) e ser sedentário (89\%). Das 148 mulheres que fizeram parte do estudo, $48 \%$ referiram já ter usado hormônios anticoncepcionais.

Analisando-se os niveis pressóricos dos 205 pacientes em estudo, a média \pm desvio padrão da pressão sistólica foi de $155 \pm 21 \mathrm{~mm} \mathrm{Hg}$ e para a diastólica $101 \pm 51 \mathrm{~mm} \mathrm{Hg}$.

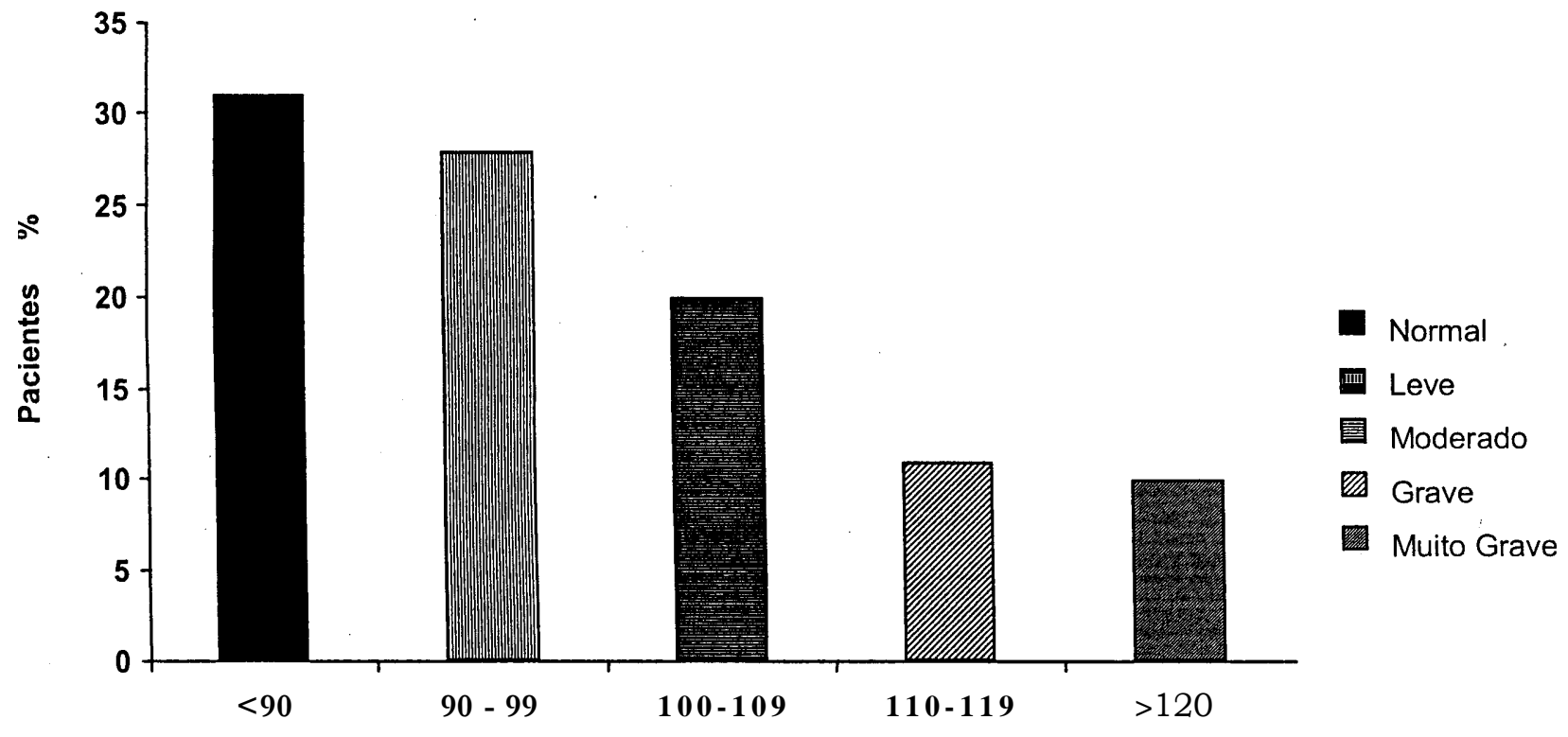

Pressão Diastólica mm Hg

Ao caracterizar os niveis da pressão diastólica os dados da Figura 1 indicam que 31\% apresentaram pressão diastólica abaixo de $90 \mathrm{~mm} \mathrm{Hg}$, portanto dentro da faixa da normalidade; $28 \%$ na faixa de hipertensão leve; $20 \%$ na faixa de hipertensão moderada; e os demais $(21 \%)$ com nível da pressão diastólica compativel com hipertensão grave ou muito grave.

\subsection{Características da história da doença dos hipertensos estudados}

A presença de antecedentes familiares para hipertensão arterial foi citada por $70 \%$ dos pacientes. Dentre estes, a maioria (66\%) apontou a mãe como o familiar com história de hipertensão na família, seguido pelo pai $(20 \%)$ e irmãos $(14 \%)$.

Em relação ao tempo de hipertensão a maior parte $(43 \%)$ sabia ser hipertensa há mais de 10 anos e $40 \%$ há 3 anos.
Tabela 2 - Distribuição do número e percentual das formas de tratamentos já realizados para Hipertensão Arterial indicadas pelos pacientes. São Paulo, 1999.

\begin{tabular}{lcc}
\hline Tratamentos & N & $\%$ \\
\hline Farmacológico & 119 & 58 \\
Não Farmacológico & 113 & 55 \\
Restrição sal & 106 & 94 \\
Restrição alimentos & 68 & 60 \\
gordurosos & 54 & 48 \\
Perda de peso & 61 & 30 \\
Outros & 49 & 81 \\
Chás & 07 & 11 \\
Homeopatia & & \\
\hline
\end{tabular}

Pouco mais da maioria dos pacientes (60\%) informou já ter realizado tratamento anti-hipertensivo. Os dados da Tabela 02 mostram que as formas mais usuais foram uso de medicamentos (58\%) e medidas não farmacológicas $(55 \%)$. Os aspectos relativos à alimentação como diminuição de sal e alimentos gordurosos foram as formas não farmacológicas de tratamento da hipertensão mais citadas. Destaca-se 
ainda que $30 \%$ referiram fazer uso de outras medidas como chás $(81 \%)$ e homeopatia $(11 \%)$.

\subsection{Caracterização do conhecimento dos hipertensos sobre a doença}

Tabela 3 - Distribuição do número e percentual dos fatores indicados pelos pacientes como associados à Hipertensão Arterial. São Paulo, 1999.

\begin{tabular}{lcc}
\hline Fatores & $\mathrm{N}$ & $\%$ \\
\hline Sal & 200 & 98 \\
Estresse & 200 & 98 \\
Bebida alcoólica & 195 & 95 \\
Fumo & 184 & 90 \\
Alimentos gordurosos & 180 & 88 \\
Excesso de peso & 165 & 81 \\
Sedentarismo & 146 & 71 \\
Aumento da idade & 135 & 65 \\
Uso de & 130 & 63 \\
anticoncepcionais & 110 & 54 \\
Hereditariedade & 41 & 20 \\
Gênero & 23 & 11 \\
Raça &
\end{tabular}

Os dados da Tabela 03 indicam que a população estudada apresentava bom nível de conhecimento dos fatores associados à hipertensão. A redução de sal na alimentação, evitar estresse, eliminar hábitos como o fumo e a bebida alcoólica foram os mais apontados. Os fatores menos indicados e, portanto, talvez menos conhecidos foram associação entre hipertensão e gênero $(20 \%)$ e com etnia $\mathbf{( 1 1 \% ) . ~ E x c e t o ~ p a r a ~ e s t e s ~}$ dois fatores todos os demais tiveram conhecimento acima de $50 \%$ da sua associação com a hipertensão arterial.

Tabela 4 - Distribuição do número e percentual de indicativos sobre a cronicidade e complicações da Hipertensão Arterial referidos pelos pacientes. São Paulo, 1999.

\begin{tabular}{lll}
\hline Indicativos & $\mathrm{N}$ & $\%$ \\
\hline Traz complicações & 201 & 98 \\
Doença grave & 185 & 90 \\
Doença incurável & 112 & 55 \\
E para toda vida & 113 & 55 \\
\hline
\end{tabular}

O conhecimento acerca dos aspectos de cronicidade e gravidade da doença apresentados nos dados da Tabela 04, indicam que 90\% consideram a doença grave e quase a totalidade (98\%) que traz complicações. Dentre as complicações foram citadas as cardiacas $(74 \%)$, "derrame" $(75 \%)$ e renais $(7,5 \%)$. Cerca da metade considerou a doença incurável e para toda vida

\subsection{O conhecimento e o perfil dos hipertensos}

O perfil dos hipertensos, identificado através da análise de correspondência, caracterizado por mulheres, cor branca, idade entre 40 e 60 anos, não casadas, obesas e que já haviam realizado tratamento anterior se associou com conhecimento sobre a doença.

Por outro lado, o perfil dos hipertensos que desconheciam aspectos sobre a doença foi formado por homens, não brancos, com idade na faixa de 20 a 40 anos, viúvos e com peso na faixa da normalidade.

\subsection{Nivel de pressão arterial e o perfil dos hipertensos.}

Para identificar possíveis associações entre o grau de hipertensão e as características da população estudada, por meio de análise de correspondência, considerou-se niveis de pressão diastólica abaixo de $110 \mathrm{~mm} \mathrm{Hg}$ (hipertensos leves e moderados) e acima ou igual a $110 \mathrm{~mm} \mathrm{Hg}$ (hipertenso grave e muito grave).

A análise dos dados revelou que os níveis mais baixos da pressão arterial (pressão diastólica $<110$ $\mathrm{mm} \mathrm{Hg}$ ) se associaram com as seguintes características: idade até 20 anos, estado civil casado, renda salarial mais que 4 salários mínimos e escolaridade equivalente ou acima do $1^{\circ}$ grau. Para os níveis mais elevados da pressão arterial (pressão diastólica $>110 \mathrm{~mm} \mathrm{Hg}$ ) o perfil da população foi para idade mais avançada (acima de 60 anos), não casados, renda inferior a 4 salários mínimos, baixa escolaridade (analfabeto ou inferior ao $1^{\circ}$ grau), na faixa de sobrepeso ou obesidade, com mais de 5 anos de doença e já ter realizado tratamento anteriormente.

\section{DISCUSSÃO}

O principal achado do presente estudo. é que homens, jovens, não brancos desconhecem aspectos importantes da doença e maiores niveis tensionais se associaram a variáveis bio sociais desfavoráveis. Considera-se que estas características possam influenciar a vivência dos hipertensos com a doença e tratamento.

$\mathrm{Na}$ caracterização da população o sexo predominante foi o feminino e idade na faixa de 41 a 60 anos. Estas duas variáveis podem influenciar o seguimento do tratamento anti-hipertensivo. Os homens tendem a ser menos aderentes, aspectos sociais e culturais, que consideram o sexo masculino na posição de comando e dominação, portanto, até isentos de doença, poderiam ser os responsáveis por esta situação. SHERBOURNE et al(10) analisaram a adesão ao tratamento em um grupo de 1198 pacientes dentre hipertensos, diabéticos e cardiacos e identificaram que pacientes mais jovens seguiam menos as recomendações médicas 
A escolaridade é um dado que também merece ser considerado. GIORGI et $\mathrm{al}^{(11)}$ em um estudo com pacientes hipertensos verificaram que houve associação entre o sexo, a idade e grau de instrução com abandono ao tratamento. Foram mais propensos ao abandono, homens com menos de 40 anos de idade e analfabetos.No presente estudo apesar do predomínio da escolaridade correspondente ao $1^{\circ}$ - grau, salienta-se que a maioria era de forma incompleta, além de pessoas analfabetas, o que caracteriza baixa escolaridade. O estado civil também deve ser destacado, considera-se que o suporte familiar oferecido não só pelo cônjuge, mas demais membros da família tenderia a apoiar o hipertenso, facilitando a sua inserção no contexto da doença e tratamento.

A raça tem papel importante na hipertensão arterial. Os negros tendem a ter níveis tensionais mais elevados do que os brancos, além de maior gravidade da doença ${ }^{(12,13)}$. Estudo avaliando a adesão em um grupo de pessoas hipertensas de raça negra revelou relação significativa entre a adesão ao tratamento e variáveis independentes como idade, educação, gênero e hábito de fumar. Os autores enfatizaram a importância de processo educativo nas escolas o mais precoce possivel quanto ao controle da pressão arterial ${ }^{(14)}$.

A condição sócio-econômica é um fator que pode influenciar na gênese e tratamento da hipertensão arterial. A falta de recursos financeiros está aliada a dificuldade de acesso aos serviços de saúde e para o cumprimento do tratamento, por exemplo na aquisição dos medicamentos. SHEA et $\mathrm{al}^{(15)}$ realizaram estudo com pessoas socialmente menos favorecidas, hispânicos e negros da cidade de Nova York, atendidas em unidades de emergência. Os resultados indicaram que os hipertensos severos não controlados atendidos em situação de emergência possuíam menor acesso aos serviços de saúde.

Hábitos como fumo e ingestão de bebida alcoólica merecem atenção na caracterização de uma população de hipertensos pela sua correlação com os niveis tensionais e, consequentemente, devendo ser afastados. Evitar fumo e bebida alcoólica juntamente com a atividade fisica regular constituem-se em elementos integrantes do tratamento não farmacológico ${ }^{(3)}$.

Portanto, as características sócio-demográficas e hábitos de vida, devem ser avaliados ao se propor ações junto às pessoas hipertensas, merecendo de todos os profissionais da área da saúde a devida atenção.

$\mathrm{Na}$ caracterização da história da doença, no presente estudo, verificou-se que $60 \%$ dos pacientes já tinham realizado tratamento anteriormente, principalmente nas formas mais usuais de tratamento farmacológico e não farmacológico, preconizadas ${ }^{(3,4)}$ Porém, ressalta-se que apesar de menos freqüente observou-se outras formas de tratamento referidas pela população, como uso de chás.

Rev.Esc.Enf.USP, v. 35, n. 1, p. 11-8, mar. 2001.
Em relação ao tratamento não farmacológico, além de cerca da metade dos pacientes apontar já têlo realizado, verificou-se nível de conhecimento bastante elevado, acima de $80 \%$ para os aspectos referentes à alimentação e excesso de peso, hábitos como fumo e bebida alcoólica e estresse da vida diária. Acredita-se que a população hipertensa deva conhecer todos os aspectos inerentes à doença e tratamento. O esclarecimento sobre fatores de risco associados, cronicidade da doença, ausência de sintomatologia específica e complicações que comprometem órgãos vitais quando não controlados os niveis tensionais, são aspectos imprescindiveis sobre os quais as pessoas hipertensas devem ser esclarecidas. Em relação ao tratamento alertar sobre suas diferentes formas e importância da continuidade para que seja efetivo. Considera-se que o processo educativo dos hipertensos constitui um fator inquestionável para o seguimento adequado do tratamento. Estudos têm revelado a importância destes

aspectos $(16,17,18)$

SADALA et al (19) realizaram estudo com o objetivo de compreender os significados atribuídos pelos hipertensos à convivência com a doença e verificaram que os pacientes apresentam visão existencial da doença: falam de sua vida, das alterações provocadas pela doença, do desconforto, do controle rígido e especialmente das emoções que devem ser evitadas. As autoras sugerem formas de oferecer aos pacientes oportunidades para superar suas dificuldades e visualizar novas possibilidades para o auto cuidado.

Cabe salientar que o fato das pessoas hipertensas estarem orientadas sobre a doença e tratamento não implica em efetivo seguimento do tratamento proposto, o que muitas vezes requer mudança de comportamento, constituindo-se um obstáculo para a adesão ao tratamento. As crenças de saúde, os aspectos culturais e comportamentais não são fáceis de manejar e modificar, requerendo de todos, paciente e grupo de saúde, devido empenho. Estudo europeu que avaliou a percepção do médico e crenças dos hipertensos a respeito do controle da pressão arterial revelou que $76 \%$ dos médicos consideraram que os hipertensos estavam controlados, contra quase a totalidade $(95 \%)$ dos pacientes, que acreditaram que a pressão arterial estava bem controlada(20)

Em nosso meio MION et al (21) realizaram estudo para avaliar as crenças e preferências em relação ao tratamento anti-hipertensivo. Os resultados mostraram que $81 \%$ desconheciam o tratamento não farmacológico, $44 \%$ tinham preferência por esta forma de tratamento e $56 \%$ pelo uso de medicamentos. Esse estudo associou também o conhecimento e preferências com o perfil dos hipertensos. Em relação ao conhecimento, nos pacientes que desconheciam o tratamento não farmacológico, destacou-se a presença 
de mulheres, de meia idade e obesas, que seriam pessoas que se beneficiariam desta modalidade de tratamento. Quanto às preferências, os pacientes mais jovens e não obesos optaram pelo tratamento não farmacológico, enquanto que os de idade mais avançada e obesos preferiram tratamento com medicamentos.

No presente estudo, analisando-se o perfil dos hipertensos e conhecimento sobre aspectos importantes da doença verificou-se que o desconhecimento se associou com o sexo masculino, não branco, jovem e viúvo. Tendo em vista que homens mais jovens podem ser mais resistentes ao tratamento, e se aliado ao desconhecimento, pode ainda mais comprometer o seguimento do tratamento. Em relação à raça, além dos aspectos de prevalência e morbi-mortalidade da hipertensão arterial serem mais contundentes na raça negra, não se pode esquecer todo o componente social envolvido nesta problemática. O negro na nossa sociedade ainda sofre um processo de desfavorecimento com sérias implicações na condição de vida, que sem dúvida alguma agrava ainda mais a condição de ser e estar doente.

No delineamento do perfil da população e associação com os niveis da pressão arterial, observou-se que para maiores niveis tensionais se associaram características de idade acima dos 60 anos, renda salarial inferior a 4 salários minimos, estado civil não casado, baixa escolaridade, peso corporal acima do normal, com mais de 5 anos de doença e realização de tratamento anterior. Considera-se que estas características induzem a aspectos desfavoráveis para a consecução do tratamento, ainda mais associadas a niveis da pressão arterial mais elevados. $\mathrm{O}$ idoso pela condição de maior comprometimento orgânico inerente ao processo de envelhecimento e escassez de assistência voltada especificamente para esta faixa etária. O estado civil não casado pode se correlacionar diretamente à falta de suporte familiar. A baixa escolaridade associada à condição financeira precária também compromete o seguimento adequado do tratamento.

Concluindo, os achados do presente estudo indicam que homens, jovens, não brancos desconhecem aspectos importantes da doença e maiores niveis tensionais se associam a variáveis bio sociais desfavoráveis. Os resultados alertam para a necessidade de se estabelecer programas de acordo com as características específicas dos hipertensos visando adesão ao tratamento.

\section{REFERÊNCIAS BIBLIOGRÁFICAS}

1. Mion Jr D, Pierin A. Causas de baixa adesão ao tratamento e o perfil de pacientes hipertensos. In: Anais do 50 Congresso da Sociedade Brasileira de Hipertensão. 1996 São Paulo, 1996. ag. 58; São Paulo. São Paulo: Sociedade Brasileira de Hipertensão; 1996. p. 120
2.Schaub 4F, Steiner A, Vetter W. Compliance to treatment. Clin Exp. Hyperten 1993;15:1121-30.

3. Sociedade Brasileira de Hipertensão. Sociedade Brasileira de Cardiologia. Sociedade Brasileira de Nefrologia. III Consenso Brasileiro de Hipertensão Arterial. Campos do Jordão SP;1998. (BG Cultural, 38)

4.National Institutes of Heath. VI Joint Nattional Committee on Prevention, Detection, Evaluation, and Treatment of High Blood Pressure. New York; 1997. (NIH Publication, 70)

5.Malachias MVB. Hipertensão Arterial em Idosos. In: Cangado FAX. Noções Práticas de Geriatria. Belo Horizonte: Coopmed; 1994. p. 269-83.

6.Pathy SJ. Hypertension and associated in elderly patients. J Hypertens 1988;6:37-40.

7.Clark LT. Improving compliance and increasing control of hypertension: needs of special hypertensive populations. Am Heart J 1993;121:664-69.

8.DiMatteo MR, Sherbourne CD, Hays RD. Physicians characteristics influence patients adherence to medical treatment: results from the medical outcomes study. Health Psycol 1993;12:93-102.

9.Klein LE. Compliance and blood pressure control. Hypertension 1988; 2 suppl 11:61-64.

10. Sherbourne CD, Hays RD, Ordway L. Antecedents of adherence to medical recommendations: results from the medical outcomes study. J Behavorial Med 1992;15:447-68.

11.Giorgi DM, Mion Jr, Car MR, Pierin AMG. Aderência do tratamento em hipertensão arterial: influência de variáveis estruturais e de estratégias que visem sua melhora. Rev Bras Med (cardiologia). 1985;4:167-75.

12. Mancilha JJC. Aspectos epidemiológicos e preventivos da hipertensão arterial. Rev Bras Clin Terap 1984;13:225-9.

13. Neaton JD. Comparison of 10 year coronary and cerebrovascular disease mortality rates by hypertensive states for black and nonblack men screned in the Multiple Risk Factor Intevention Triol (MRFIT). Circulation suppl II 1989;80:II-300.

14.Daniels DE, Rene AS, Daniels VR. Race: an explanation of patients compliance - factor or fiction? J Natl. Med. Assoc 1994;86:20-5.

15.Shea S, Mirra D, Ehrlich MH, Field L, Francis CK. Predisposing factors for severe, uncontrolled hypertension in an inner-city minority population. N Engl J Med 1992;327: 776-81.

16. Car MR, Pierin AMG, Aquino VLA. Estudo sobre a influência do processo educativo no controle da hipertensão arterial. Rev Esc Enf USP 1991;25:259-69.

17.Darine EC, Reifschneider A. A meta- analyses of the effects of psychological care in adults with hypertension. Nurs Res 1995;44:37-45..

18.DiMatteo MR, Sherbourne CD, Hays RD. Physicians characteristics influence patients adherence to medical treatment: results from the medical outcomes study. Health Psycol 1993;12:93-102.

19.Sadala, M.L; Mendes, I. M. Vivenciando a hipertensão. Rev Soc Cardiol Est São Paulo 1996; supl A 3:1-6.

20.Hosie J, Wiklund I. Managing hypertension in general practice: can we do better? J Hum Hypertens 1995; suppl 2P 9:515-18.

21.Mion Jr D, Pierin A, Ignes E, Ballas D, Marcondes $\mathrm{M}$. Conhecimentos, preferências: o perfil dos hipertensos quanto ao tratamento farmacológico e não farmacológicos. J Bras Nefrol 1995; 17:229-36. 


\section{Instrumento de coleta de dados (Anexo 1)}

\section{IDENTIFICAÇÃO}

Nome:

Idade:

Sexo:

RG:

Pressão arterial

Peso

Altura IMC

Estado civil: ( ) casado

$\mathrm{mm} \mathrm{Hg}$

FC:
( ) viúvo
() solteiro
() separado
() outro

Cor: () branca

() negra

() mulata

() amarela

Escolaridade: ( ) sabe ler/ escrever
( ) primeiro grau
( ) completo
() segundo grau
( ) incompleto
() superior
() analfabeto

Renda salarial: ( ) < 1 salário mínimo

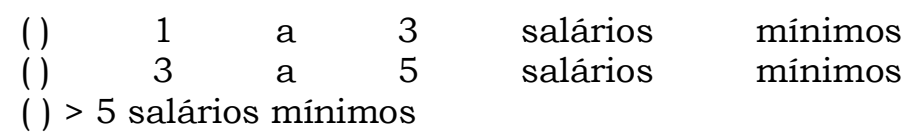

Bebida alcóolica: ( ) sim
()bebeu anteriormente
( ) não

Fumo: ( ) sim

()fumou anteriormente

( ) não

Uso de hormônio anticoncepcional: ( ) sim
( ) usou anteriormente
( ) não

Exercícios físicos: ( ) sim

qual:
( ) fazia anteriormente
qual:
( ) não

Antecedentes de hipertensão na família: ( ) sim quem ( ) não

Faz tratamento com medicamentos para hipertensão arterial
( ) não
() $\operatorname{sim}$

Rev.Esc.Enf.USP, v. 35, n. 1, p. 11-8, mar. 2001. 


\section{Faz outros tipos de tratamento para hipertensão arterial}

( ) não

( ) $\operatorname{sim}$

Quais?

Quais dos fatores relacionados a seguir você acha que pode estar relacionado com hipertensão arterial? ( assinalar na coluna correspondente)

\begin{tabular}{l|l|l}
\hline Fatores & Sim & Não \\
\hline Sal & & \\
Stress & & \\
Bebida alcoólica & & \\
Fumo & & \\
Alimentos gordurosos Excesso de peso & \\
Falta de atividade fisica Aumento da idade & & \\
Uso de anticoncepcionais Hereditariedade & & \\
Sexo da pessoa & & \\
Raça & & \\
\hline
\end{tabular}

Você acha que pressão alta traz complicações?

$$
\begin{aligned}
& \text { ( ) Sim } \\
& \text { ( ) Não }
\end{aligned}
$$

Você acha que pressão alta é para toda vida?

$$
\begin{aligned}
& \text { ( ) Sim } \\
& \text { ( ) Não }
\end{aligned}
$$

Você acha que pressão alta tem cura?

$$
\begin{aligned}
& \text { ( ) Sim } \\
& \text { ( ) Não }
\end{aligned}
$$

Você acha que pressão alta é uma doença grave ?

$$
\begin{aligned}
& \text { ( ) Sim } \\
& \text { ( ) Não }
\end{aligned}
$$

